\title{
Die Osterweiterung der EU aus der Sicht bestehender Mitgliedsländer: Was lehrt uns die Theorie der ökonomischen Integration?
}

von

Wilhelm Kohler

Arbeitspapier Nr. 0001

Januar 2000

Erscheint in

Perspektiven der Wirtschaftspolitik

Institut für Volkswirtschaftslehre

Johannes Kepler Universität Linz A-4040 LINZ - AUHOF

Tel.: 00437322468 237, Fax -238

e-mail: wilhelm.kohler@jk.uni-linz.ac.at

http://www.economics.uni-linz.ac.at

\footnotetext{
* Diese Arbeit basiert auf einem Plenarvortrag bei der Jahrestagung des Vereins für Socialpolitik, 28.9.-1.10.1999 in Mainz. Gabriel Felbermayr gebührt Dank für sehr wertvolle Hilfe bei den empirischen Berechnungen, und Wolfgang Leitner für die Aufbereitung der Tabellen und Graphiken.
} 


\section{Einleitung}

Nach dem Zusammenbruch der politischen und wirtschaftlichen Systeme der Länder Mittel- und O steuropas schien deren Eintritt in die bestehenden Integrationsstrukturen Westeuropas die natürlichste Sache der Welt. Die E uropäische U nion reagierte denn auch relativ schnell, und zwar zunächst durch den Abschluß von bilateralen Abkommen über die wechselseitige Beseitigung der formalen Handelsbarrieren, den sogenannten E uropa A bk ommen, und dann durch die prinzipielle Öffnung der Gemeinschaft in Richtung Osten. Die vertraglichen Grundlagen dafür wurden in Maastricht geschaffen, und das politische Bekenntnis zur Osterweiterung erfolgte durch den Ratsbeschluß beim Kopenhagener Gipfel im Jahre 1993. Von Anfang an konnte man sich allerdings des Eindrucks eines gewissen Interessengegensatzes nicht erwehren. Während die Vollmitgliedschaft den Ländern Mittel- und O steuropas nicht schnell genug kommen konnte, wurde in den bestehenden Mitgliedsländern sehr schnell die Sorge vor einer zu raschen Erweiterung geäußert. Schon 1994 sah sich die EU mit den ersten formellen Beitrittsansuchen konfrontiert, aber es sollte bis 1998 dauem, ehe die ersten 5 Länder an den Verhandlungstisch gebeten wurden (Estland, Polen, Slowenien, Tschechische Republik und Ungam). Und die restlichen 5 Beitrittswerber (Bulgarien, Lettland, Litauen, Rumänien und die Slowakische Republik) mußten bis zum Gipfel von Helsinki - 10 Jahre nach dem Fall des Eisernen Vorhangs - auf eine Öffnung der Tür zu konkreten Beitrittsverhandlungen warten.

Warum diese Zähigkeit? Die EU hat stets betont, daß ein Beitritt nur im Wege einer Übernahme und glaubwürdigen Implementierung des gesamten $\mathrm{G}$ emensamen Rechtsbestandes der Union („acquis communautaire") erfolgen könne. Dies zu gewährleisten, ist Zweck der Beitrittsverhandlungen, und nachdem es sich dabei um einen nicht weniger als 80.000 Seiten umfassenden Kodex handelt, scheint die langsame Geschwindigkeit nicht weiter überraschend. Das ist die wohlwollende Interpretation. Indes ist die Umsetzung des „acquis“ nicht ein objektiv meßbarer Sachverhalt, vielmehr ist dabei ein genüttelt Maß an Interpretation und Freiraum im Spiel. Einer anderen, etwas weniger freundlichen Interpretation zufolge ist der "acquis" ein dehnbares Instrument, mit Hilfe dessen die Union die O sterweiterung, zu der sie sich nun einmal im Prinzip und unwiderruflich bekannt hat, in zeitlicher Hinsicht gestalten, d.h. bei Bedarf eben auch verzögern kann. Nicht die Sorge um die mangelnde Umsetzung des "acquis" und die damit verbundenen Probleme der Anpassung in den neuen Mitgliedsländem sind nach dieser Interpretation die eigentlich retardierenden Elemente, vielmehr ist es die E rwartung negativer A uswirkungen der Erweiterung auf die bestehenden Mitgliedsländer. Im Unterschied zur vierten Erweiterung sollen nun Länder beitreten, die nach geltender EU-Politik N ettoempfänger von E U. Mitten sein werden. Hinzu kommt, daß die Auswirkungen der regionalen A usdehnung des Integrationsrau- 
mes in den bestehenden Mitgliedsländem durchaus nicht einmütig als positiv empfunden werden. Auf diese Weise kann sich die O sterweiterung aus westlicher Sicht sehr leicht als Frage mehr der politischen Räson, oder gar der moralischen Pflicht, denn des ök onomischen $V$ orteils darstellen. Ist sie gar nichts anderes als der Preis, den die westlichen Länder für die Fortsetzung der Reformbestrebungen in den ehemals sozialistischen Nachbarländern zu zahlen haben?

Nun darf aber die Frage nach den ökonomischen Auswirkungen der O sterweiterung für die bestehenden Mitgliedsländer nicht Tabu sein. Wichtig ist, daß die Antwort auf solider theoretischer Grundlage und unter Anwendung explizit gemachter Kriterien gesucht wird. Wir haben es hier mit einem klassischen A nwendungsfall der Theorie der ök onomischen Integration zu tun. D iese hat ein umfangreiches Instrumentarium zur Identifikation von Vorteilen und Nachteilen der Errichtung und Ausdehnung von regionalen Integrationsblöcken nach dem Muster der EU entwickelt. Es scheint angezeigt, nach Möglichkeiten der Anwendung dieses Instrumentariums zu suchen, um die weitgehend und einseitig von der Sorge über die Nettotransfers an die neuen Mitgliedsländer dominierte Sicht der Osterweiterung auf eine etwas breitere $G$ rundlage zu stellen. D ies soll hier geschehen. Es folgen zunächst grundsätzliche Anmerkungen zur Frage der Liberalisierung, danach werde ich für jedes der bestehenden EU-Mitgliedsländer einige empirische Berechnungen zu den Liberalisierungseffekten präsentieren. Diese werden zur Abrundung des Bildes ergänzt durch einen Blick auf die fiskalischen Belastungen, welche die Länder aufgrund der Nettotransfers an die neuen Mitglieder zu erwarten haben. Auf diese Weise sollen Anhaltspunkte dafür gefunden werden, welche Länder von der Osterweiterung ökonomische Vorteile bzw. Nachteile zu erwarten haben.

\section{Grundsätzliches zur Frage der Liberalisierung}

Die O sterweiterung der EU beinhaltet weit mehr als einen bloßen Schritt der Liberalisierung, selbst wenn man, wie ich das hier tun möchte, die Europa Abkommen über die Beseitigung der formalen Handelsbarnieren als integralen Bestandteil der Erweiterung betrachtet. Das gilt insbesondere für neue Mitgliedsländer, für die der erwähnte „acquis“ in fast allen Bereichen des ökonomischen Lebens fundamentale, und nicht selten auch schmerzliche Veränderungen bereithält. Für die bestehenden Mitgliedsländer besteht die Erweiterung indes aus drei relativ leicht umschreibbaren Veränderungen: a) der Liberalisierung, bestehend aus der Ausdehnung der Zollunion und des Gemeinsamen Marktes („Single Market") auf die neuen Partnerländer, b) der fiskalischen Belastung, die aus der Anwendung der EUAgrar- und -strukturpolitik auf die neuen Mitglieder resultiert, sowie c) einer institutionellen Veränderung. Letztere beinhaltet nicht nur eine veränderte Stimmengewichtung im Europäischen Rat, dem zentralen Entscheidungsgremium der EU, sondern eine weit darüber hinausgehende Reform, welche 
die institutionelle Struktur der G emeinschaft mit der neu erreichten Größe in Übereinstimmung bringen soll. Dies wird G egenstand der kommenden Regierungskonferenz sein, und ich werde mich dazu hier im Weiteren nicht äußern. Bezüglich der Punkte a) und b) beobachtet man eine ausgeprägte Asymmetrie. Die fiskalischen Implikationen der Erweitenung (Punkt b) werfen kaum prinzipielle Verständnisfragen auf, sie stellen in erster Linie ein empirisches Problem dar, auf das ich später zurück kommen werde. Im Unterschied dazu sehen wir uns bei den Liberalisierungswirkungen (Punkt a) mit grundsätzlichen Verständnisproblemen konfrontiert, die ich vorab erörtern möchte.

\section{Fehlgeleitete Prinzipien}

Die öffentlichen Diskussionen über die internationale Liberalisierung von Güter- und Faktormärkten, wie auch die Prinzipien, denen die praktizierte Politik zu folgen scheint, nehmen in einem für Ökonomen oft frustrierend geringen Ausmaß Bezug auf etablierte Erkenntnisse der ökonomischen Theorie. Krugman (1991a) beschreibt drei Leitideen, die in der Praxis dominieren, ökonomisch betrachtet aber absolut keinen Sinn machen: 1) „Exporte sind gut", 2) „Importe sind schlecht", und 3) "ceteris paribus geht es darum, eine gleich starke Zunahme von Importen und Exporten anzustreben“. Er nennt diese Ideen "G A TT - think", heute würden wir wohl „W T0 - think" sagen, aber sie sind auf nicht minder prägende Weise auch präsent bei regional begrenzten, d.h. präferenziellen Liberalisierungsschritten, wie etwa im Fall der Erweiterung der EU.

Der ökonomische Unsinn dieser Prinzipien ist eigentlich schnell erkannt. Der Vorteil internationaler Wirtschaftsbeziehungen resultiert aus A rbeitstelung, also aus Tausch von exportierten Gütern gegen Importe, wo das eine ohne das andere nicht vorstellbar ist. Fügt man dem noch die Vorteile des intertemporalen Tausches hinzu, der zwingend mit temporär unausgeglichenen Leistungsbilanzen einher geht, so wird auch dem Prinzip 3 die Grundlage entzogen. Es ist nicht überraschend, daß derart fehlgeleitete Prinzipien in der Praxis genau jenes Prinzip des internationalen Handelssystems ausgehöhlt haben, das als einziges auch unmittelbar ökonomisch Sinn macht, nämlich das des M ultilateralismus. Im Rahmen der WTO ist dieses Prinzip verankert im Diskriminierungsverbot. Dieses Prinzip besagt in unserem Zusammenhang, daß Liberalisierung gegenüber allen Ländern praktiziert wird, und nicht nur eingeschränkt auf einen Block von Ländem, die aus irgendwelchen G ründen als Partnerländer präferiert werden. Man spricht in diesem letzteren Falle von präferenzieller Liberalisierung. Wie unten noch klar werden wird, birgt präferenzielle Liberalisierung die $\mathrm{G}$ efahr einer schädlichen $\mathrm{H}$ andesumlenk ung.

Dessen ungeachtet haben in den vergangenen 15 Jahren viele Länder verstärkt auf regionale Integrationsblöcke gesetzt, und zwar in konsequenter Verfolgung der obigen Prinzipien 1-3. Diese führen nämlich nahtlos zu einer Strategie des „do ut des“, wonach die Öffnung der heimischen Märkte immer als „Konzession“ verstanden wird, als G egenleistung für die den garantierten Zugang zu Exportmärk- 
ten. Dieses Prinzip hat in das Regelwerk des GATT sogar formell Eingang gefunden, und zwar unter dem schön klingenden Namen Reziprozität. Aber es zeigte sich eben, daß „do ut des“ mit einer beschränkten Menge von Partnerländem leichter realisiert werden kann, als in multilateralen Verhandlungen. So ist das Prinzip des Multilateralismus in Konflikt geraten mit jenem der Reziprozität, und das letztere hat - zumindest temporär - die O berhand gewonnen. Und wieder ist es das falsche, denn Reziprozität ist im Lichte der ökonomischen Theorie zunächst nicht minder fehlgeleitet als die Prinzipien 1-3. Warum soll sich ein Land der Vorteile des Tausches begeben, bloß weil die anderen Länder es vorziehen, dies nicht zu tun?

Warum betone ich all dies im Zusammenhang mit der O sterweiterung der EU? Aus drei G ründen. Erstens macht es deutlich, daß beim oben erwähnten Punkt b) die Vorteile der Liberalisierung nicht einseitig in neuen Exportmöglichkeiten gesucht werden dürfen. Sie liegen in neuen und frik tionsfreieren Tauschmöglidk eiten, und dies betrifft freilich auch die Importseite. Zweitens wird uns dadurch bewußt, daß die mit der O sterweiterung einher gehende Liberalisierung des Handels aufgrund ihrer präferenziellen $\mathrm{N}$ atur das Prinzip des Multilateralismus durchbricht, und deswegen kein einwandfreier Schritt der Liberalisierung darstellt. Nicht daß damit das WTO Reglement verletzt würde, denn dieses sieht im Artikel XXIV explizit eine Ausnahme für regional begrenzte Integrationsblöcke vor. Und die EU als solche ist schon seit langem in diesem Sinne als "legitime Ausnahme" akzeptiert. Der Einwand ist vielmehr der, daß die regionale Präferenz der Liberalisierung immer neue Verzerrungen bedingt, die ihrerseits Handelsumlenkungen von D rittländem zu den neuen Mitgliedsländem zur Folge haben können, welche für bestehende Mitgliedsländer im Sinne des Punktes b) zu Wohlstandseinbußen führen. Drittens aber erhebt sich die Frage, ob die Osterweiterung der EU das Welthandelssystem als solches auf signifikante Weise verändem wird. Damit ist die Frage nach den systemischen A uswirkungen in Kontext der Debatte um „Regionalismus" versus „Multilateralismus" gestellt. Konkreter formuliert: Wird die O sterweiterung der EU, ohne Z weifel ein „regionalistischer" Schritt, das multilaterale Handelssystem auf einen vom Multilateralismus wegführenden, und insgesamt weniger attraktiven Entwicklungspfad führen? Eine ins D etail gehende Erörterung dieser Frage würde vom Thema wegführen, aber ein paar Bemerkungen sind gleichwohl angebracht.

\footnotetext{
${ }^{1}$ Dieser Punkt wird manchmal durch die recht anschauliche Parabel eines Landes verdeutlicht, das seine Hafenmauem niederreißt, bloß weil die anderen Länder felsige Küsten haben; siehe z.B. Krugman (1991a).

2 Ausführlichere Abhandlungen zu diesem Problem finden sich vor allem in Bhagwati (1993), Bhagwati \& Panagariya (1996), sowie Bhagwati et al. (1998), und Ethier (1998).
} 


\section{Bestärkt die Osterweitenung den „neuen Regionalismus“?}

Zunächst könnte man versucht sein, in der O sterweiterung einen Schritt in Richtung jener „3Blöcke-Welt" sehen, die Krugman (1991b) als schlechteste aller O ptionen skizziert hat. Aber liefe wohl auf eine nahezu groteske Überstrapazierung des von Krugman zugrunde gelegten Modells hinaus. Etwas moderater, aber im Grunde in dieselbe Richtung blickend, kann man sich fragen, wodurch das Endziel eines von künstlichen Barrieren befreiten Welthandels besser erreicht werden kann: auf dem Weg des Multilateralismus der WTO, oder auf dem Weg des „neuen Regionalismus“, paradigmatisch repräsentiert durch die Osterweiterung der EU? ${ }^{\text {T }}$ D ies ist die von Bhagwati (1993) aufgeworfene Z eitpfad-F rage. Es geht hier letztlich um zwei Kategorien von Anreizmechanismen. Zum einen den Anreiz zu einer weiteren Ausdehnung existierender Blöcke, zum anderen um den Anreiz zum Engagement der in Integrationsgebilden organisierten Länder (bzw. der Blöcke selbst) für multilaterale Liberalisierungsbestrebungen. Je stärker diese Anreize, um so weniger abträglich ist der präferenzielle Weg für den Multilateralismus. Im Fall der EU wirken die ex ternen Anreizsstrukturen offenbar in Richtung Erweiterung; an Beitrittswünschen mangelt es ja nicht. Und Baldwins (1996) „D omino Theorie“ des „neuen Regionalismus" läßt erwarten, daß diese Anreize zu weiteren Beitrittswünschen führen werden. Zugleich aber zeigen die Diskussionen in den bestehenden Mitgliedsländern deutlich, daß die internen Anreize eher retardierender Natur sind. Man braucht nicht viel Phantasie, um sich die momentanen Mitgliedsaspiranten bei künftigen Erweiterungsrunden als bremsende Kräfte vorzustellen. Was das Engagement für multilaterale Liberalisierungsbemühungen anlangt, so gibt einerseits die theoretische Forschung Anlaß zu Skepsis, ${ }^{5}$ andererseits aber kann man festhalten, daß die fraglichen Länder mit einem EU-Beitritt ja nicht aus dem multilateralen Handelssystem ausscheren. Vielmehr sind sie - mit Ausnahme Estlands und Litauens - allesamt bereits Mitglieder der WTO, ebenso wie die EU als Ganzes.

\section{Die „polit-ökonomische“ Perspektive}

Man kann die systemische Frage allerdings auch unter einem anderen Gesichtspunkt betrachten. Wenn oben gesagt wurde, die leitenden Prinzipien praktizierter Handelspolitik seien - mit Ausnahme

\footnotetext{
${ }^{3}$ Siehe dazu auch Krugman (1993), sowie vor allem D eardorff \& Stern (1994).

${ }^{4}$ Die Wesensmerkmale des „neuen Regionalismus“ sind: a) Die Anbindung relativ kleiner Länder an bereits existierende, große Blöcke, b) die gleichzeitige (oder vorangegangene) Reform dieser Länder in Richtung des „ökonomischen Modells“ der Blockländer, und c) eine über die bloße Beseitigung von formalen Handelsbamieren hinausgehende Integration von Güter- und Faktormärkten. Siehe dazu insbesondere Ethier (1998).

${ }^{5}$ Siehe dazu z.B. Krishna (1998) und Levy (1997).
} 
des Multilateralismus - ökonomischer Unfug, so geschah dies aus der Warte des „wohlmeinenden Diktators“. Aus der Warte der „neuen politischen Ökonomie“ sieht die Sache anders aus. In der Tat muß man ja feststellen, daß der GATT/ WTO Ansatz zur Handelspolitik, sieht man einmal von dem auf spektakuläre Weise fehlgeschlagenen Versuch einer "Millennium-Verhandlungsrunde" in Seattle ab, gar nicht so schlecht funktioniert hat. Dies hat damit zu tun, daß Regierungen typischerweise nicht „wohlmeinende Diktatoren“ sind, die an den „gains from trade“ für ihre Länder interessiert sind. Vielmehr stehen sie unter dem D ruck von partikulären Interessen, die auf höchst unterschiedliche organisiert sind. D ie Prinzipien 1 und 2 reflektieren den Umstand, daß die hinter Exportsteigenungen stehenden G ruppeninteressen im politischen Prozeß ein größeres $G$ ewicht haben als die G ruppe jener, die an billigen Importen interessiert ist. Wären die Regierungen jeweils unabhängig voneinander diesem asymmetrischen D ruck ausgesetzt, so entstünde eine Art Pattsituation, in der sie sich wechselseitig an der Realisierung jedweder Vorteile aus intemationalem Handel behindem. Das GATT-Prinzip der Reziprozität verbindet nun die Länder in der Weise miteinander, daß die Exportinteressen des einen Landes im Rahmen von Verhandlungen zu einem Gegengewicht werden für die anti-Import-Interessen des anderen Landes. Dies gilt jedenfalls solange, als es gelingt, die Verfolgung der Exportinteressen mit Hilfe von Exportsubventionen zu unterbinden, wie das im GATT/ WTO-Reglement mehr oder weniger erfolgreich versucht wird!

Die hier nur grob skizzierte Idee, daß unter dem D ruck von heimischen Interessengruppen agierende Regierungen einander wechselseitig Schaden zufügen, ist von Bagwell \& Staiger $(1998,1999)$ zu einer ökonomischen Theorie des GATT (der WTO) formalisiert worden, die nicht nur dem Prinzip der Nichtdiskriminierung, sondem auch dem Prinzip der Reziprozität einen positiven Wert beimißt. Bagwell und Staiger gehen davon aus, daß die Regierungen den intemationalen Handel als Aktionsfeld für Interventionen begreifen, mit denen verteilungspolitische Ziele, und damit letztlich politische Macht erreicht bzw. erhalten werden kann. Sie zeigen, daß dies unter sehr allgemeinen Bedingungen mit einer gezielten Beeinflussung der Terms-of-trade, der relativen Preise im internationalen Handel, einher geht. Was nun aber aus der Sicht des einen Landes eine Terms-of-trade Verbesserung darstellt not ebne: polit-ökonomisch, nicht traditionell normativ betrachtet -, das bedeutet mitunter eine Terms-of-trade Verschlechterung für ein anderes Land. Ein effizienter Zustand wäre dann erreicht,

${ }^{6}$ Siehe dazu auch Krugman (1991a). Man kann das GATT bzw. die WTO so gesehen durchaus als „Pakt mit dem Teufel", hier konkret in Gestalt des Merkantilismus, sehen. Dies funktionierte so lange recht gut, als die handelnden Personen sich dieses Umstandes bewußt waren. Neuerdings sind hier jedoch Zweifel angebracht. Mehr und mehr gewinnt man den Eindruck, daß die handelnden Personen sich den erwähnten merkantilistischen Grundsätzen um ihrer selbst Willen verschrieben haben, so daß aus dem „Pakt mit dem Teufel“ innere Überzeugung geworden ist. 
wenn jedes Land bei seinen Maßnahmen diese externen Effekte auf andere Länder mit berücksichtigt, also wenn sie internalisiert würden. Auf diese Weise würden die Länder ihre verteilungsorientierten Ziele auf Pareto-effiziente Weise emeichen können. Das Kernergebnis dieser Analyse ist, daß die in der WTO praktizierten Prinzipien der Nichtdiskriminierung und Reziprozität solcherart effiziente Zustände als Gleichgewichte zu stützen vermögen.

Damit werden die systemischen Kosten der Duchkreuzung des GATT/ WTO Ansatzes durch den „neuen Regionalismus" auf theoretischer Ebene, d.h. über bloße Plausibilität hinaus, konkret benannt. Was bleibt, ist die Frage nach der relativen Bedeutung dieser Kosten im konkreten historischen Kontext. Unstrittig ist, daß die EU ein präferenzielles Integrationsgebilde darstellt, das dem Prinzip des Multilateralismus widerspricht. Ebenso unstrittig ist, daß die EU in Sachen Protektionismus keine reine Weste hat. Die historisch relevanten Fragen aber sind hier eigentlich andere, nämlich - erstens - ob eine nach O sten erweiterte EU im Konzert der WTO eine weniger „rühmliche“ Rolle spielen wird als die EU15 es tun würde, und - zweitens - ob die ehemals sozialistischen Länder Mittel- und O steuropas, wären sie keine EU-Mitglieder, sich in der WTO kooperativer verhalten würden, denn als Mitglieder der EU. Beides scheint sehr zweifelhaft, und darüber hinaus scheinen diese Fragen angesichts anderer Probleme, mit denen die WTO derzeit konfrontiert ist, schlichtweg marginal.

Nun kann man allerdings internationale Abkommen nicht nur als Mittel des internationalen Interessenausgleichs begreifen, sondern auch als Instrument der Absicherung eines wirtschaftspolitischen Programms. Krugman (1993) sieht den eigentlichen Zweck internationaler Handelsabkommen sogar darin, die Länder jeweils vor den internen Interessengruppen zu schützen, und nicht vor den Interessen anderer Länder. D amit wird die eben skizzierte polit-ökonomische Perspektive im Prinzip aufrecht erhalten, aber die Argumentationsrichtung wird gewissermaßen auf den Kopf gestellt. Es wurde oft darauf hingewiesen, daß der Beitritt zu regionalen oder multilateralen Handelsabkommen gerade für Reformländer von großem Vorteil sein kann, und zwar im Sinne eines Instruments der glaubwürdigen Bindung. Ethier (1998) betont, daß dies gerade beim „neuen Regionalismus“ ein bedeutender Punkt ist. Und hier muß man nun ohne Zweifel feststellen, daß dieser Vorteil für die Länder Mittel- und O steuropas bei einem EU-Beitritt größer ist, als bei einem Beitritt zur WTO allein. Man denke hier nur an die ungleich stärkeren Durchsetzungs- und Sanktionsmechanismen der EU. Alles in allem kann man also wohl festhalten, daß die O sterweiterung der EU trotz der präferenziellen Natur der damit verbundenen Liberalisierung nicht zu einer signifikanten Unterminierung des multilateralen Handelssystems führen wird. 


\section{Die Liberalisierungseffekte: Ein Versuch der Q uantifizierung}

Regierungen mögen durch polit-ökonomische Motive geleitet werden, aber das sollte die Ökonomen nicht daran hindern, normative Überlegungen der traditionellen Art anzustellen, und die Regierungen nach Möglichkeit auch mit quantitativer Information betreffend die gesamtwirtschaftlichen Kosten ineffizienter Politiken bzw. die Vorteile effizienzsteigemder Politiken zu konfrontieren. In diesem Sinne tut es sicherlich not, einen Blick auf die Liberalisierungseffekte der Osterweiterung zu werfen.

\section{E ine einfache Betrachtung: „natürliche“}

Die O sterweiterung der EU stellt sich für ein Land am Westrand der EU klarerweise anders dar als für Länder in unmittelbarer Nachbarschaft zu den neuen Mitgliedsländern aus dem O sten. Die Tabelle 1 präsentiert den Handel verschiedener EU15-Länder mit MOEL, jeweils ausgedrückt in Prozent des BIP. Wir beobachten ein durchaus differenziertes Muster der Exponiertheit gegenüber den Liberalisierungseffekten der Erweiterung. Nach den oben erwähnten Prinzipien der Liberalisienungspolitik würde man die Exportanteile als Maß für erhoffte Vorteile und die Importanteile als Bedrohungspotential interpretieren. Ich habe schon betont, daß diese Interpretation fehlgeleitet ist. Weniger problematisch scheint es hingegen, diese Anteile als Maß dafür zu sehen, wie „natürlich“ die neuen Mitgliedsländer als Handelspartner für die bestehenden Mitgliedsländer sind, wie bedeutend demnach die Ausdehnung der Zollunion und des Gemeinsamen Marktes für die diese Länder ist. Dies knüpft an die von Krugman (1991a) und Summers (1991) propagierte Vorstellung, daß Länder mit intensiven Handelsbeziehungen in dem Sinne „natürliche“ Kandidaten für präferenzielle Integrationsblöcke darstellen, daß die positiven Handelsschaffungseffekte die schädlichen Handelsumlenkungseffekte dominieren. Das Argument ist allerdings von den überzeugten „Multilateralisten“ vehement kritisiert worden, insbesondere von Bhagwati und Panagariya. ${ }^{\square}$ Die Kritik richtet sich insbesondere gegen die leichtfertige Berufung auf dieses Kriterium zwecks Rechtfertigung bestimmter präferenzieller Integrationsschritte bei (angeblich) gleichzeitiger Anerkennung der prinzipiellen Überlegenheit des mutlilateralen Systems. Für unsere Z wecke ist allerdings schwer von der Hand zu weisen, daß die O sterweiterung für Länder mit geringen Export- und/ oder Importanteilen in puncto Liberalisierungseffekte weniger bedeutsam ist als für Länder mit hohen Anteilen. Zugleich aber erkennt man schnell, daß das durch die Tabelle 1 gezeichnete Bild noch viel zu grob ist, um daraus Schlußfolgerungen ziehen zu können, die im Sinne der einleitenden Bemerkungen den fiskalischen Belastungen der Osterweiterung gegenübergestellt werden

\footnotetext{
${ }^{7}$ Siehe Bhagwati (1993), Bhagwati \& Panagariya (1997), sowie Bhagwati, G reenaway \& Panagariya (1998).
} 
können (Punkte a und b). D afür ist eine differenziertere Betrachtung auf der Grundlage der Integrationstheorie vonnöten.

\section{Differenziertere Betrachtung: „Wohlstandsdreiecke“ und „Wohlstandsrechtecke"}

Die moderne Theorie identifiziert mehrere Wirkungskanäle von regional begrenzten Integrationsschritten wie der O sterweiterung der EU. Eine übersichtliche Darstellung findet man in Baldwin \& Venables (1995); Panagariya (1997) bietet eine moderne Präsentation der traditionellen Handelseffekte, und zwar unter Anknüpfung an das eben erwähnte Argument der „natürlichen“ Handelspartner. In Kohler (1999) werden diese Effekte in einem allgemein formulierten Modell mit der Osterweiterung der EU in Verbindung gebracht. Hier möchte ich mich auf eine vereinfachte graphische Darstellung beschränken, auf deren Grundlage ich später einige Berechnungen präsentieren werde.

Die Abbildung 1 zeigt zwei Importnachfragefunktionen eines bestehenden EU Landes, die linke für ein Gut $O$ aus MOEL, die rechte für ein konkurrierendes Gut $R$ aus dem Rest der Welt. ${ }^{[} \mathrm{D}$ iese Funktionen geben für alternative Importmengen die mit zunehmender Nachfrage abnehmende marginale Zahlungsbereitschaft der Nachfrager an, und zwar ausgedrückt in Einheiten eines beliebigen heimischen Gutes, für das Marktverzerrungen existieren. Durch einen Importzoll werden die Konsumentscheidungen verzent, da ein Teil der Zahlungsbereitschaft gewissermaßen durch den Zoll „abgeschöpft" wird. Dies wird durch die um den Faktor $1 /\left(1+t_{U}\right)$ nach unten verschobenen Nachfragefunktionen $N_{O}^{t}$ bzw. $N_{R}^{0 t}$ angedeutet, wobei $t_{U}$ den gemeinsamen EU-Außenzoll darstellt. Der Einfachheit halber nehmen wir an, die Güter werden von den ausländischen Produzenten zu konstanten Preisen $\bar{p}_{O}$ bzw. $\bar{p}_{R}$ angeboten. Aufgrund von kostspieligen technischen Handelsbarrieren ${ }^{\mathbb{E}}$ werden die Güter „an der Grenze“ zu einem über $\bar{p}_{O}$ bzw. $\bar{p}_{R}$ liegenden Preis verfügbar. Wir nennen diese Differenz hier einfach die realen Transaktionsk osten der Importe. Unter Berücksichtigung der Zollbelastung kommt es vor der O sterweiterung zu Importmengen $M_{O}^{0}$ bzw. $M_{R}^{0}$, bei inländischen Konsumentenpreisen $p_{O}^{0}$ bzw. $p_{R}^{0}$. Man beachte, daß die Nachfragefunktionen jeweils auch vom Preis des anderen Gutes abhängen. Die Linie $N_{O}$ für das Gut $O$ gelte für den Preis $p_{R}^{0}$ des Gutes $R$, und umgekehrt die Linie $N_{R}^{0}$ für den Preis $p_{O}^{0}$ des Gutes $O$. Nun kommt es im Zuge der Osterweiterung

\footnotetext{
8 Die Darstellung ist zu illustrativen Zwecken stark vereinfacht. Sie ignoriert jegliche Einkommenseffekte, und sie beschränkt sich auf Kreuzpreiseffekte zwischen zwei konkurrierenden Gütern aus MOEL und aus D rittländern.

${ }^{9}$ Gemeint sind hier vor allem Grenzkontrollen, die Beibringung von Ursprungszeugnissen und Zertifikaten, sowie die Anpassung an fremde Produktestandards; siehe dazu Leonhard (1998) und Brenton (1999)
} 
zu einer Ausdehnung der Zollunion und des gemeinsamen Marktes. Es verschwinden in der linken Hälfte der Darstellung sowohl der Zollsatz, als auch die realen Transaktionskosten, in der rechten Hälfte hingegen bleiben sie bestehen. Das ist es, was mit der präferenziellen Natur der Liberalisierung gemeint ist. Es kommt zu einer Ausdehnung der Importe aus MOEL auf das Niveau $M_{O}^{1}$, da diese nun im Inland zu einem von Barrieren befreiten Preis $\bar{p}_{O}$ verfügbar werden. Diese Verbilligung verschiebt aber zugleich die Nachfragefunktion für Güter aus D rittländern von $N_{R}^{0}$ auf die Position $N_{R}^{1}$ bzw. - unter Berücksichtigung des Zollsatzes - auf $N_{R}^{1 t}$. Es kommt bei unverändertem Preis zu einer Reduktion dieser Importe auf das Niveau $M_{R}^{1}$. Worin bestehen nun die Wohlstandswirkungen dieser Veränderungen?

1. H andelsschaffung: Bei der Bewegung entlang der Linie $N_{O}$ von $M_{O}^{0}$ zu $M_{O}^{1}$ werden zusätzliche Güter importiert, für welche die Zahlungsbereitschaft im Inland die Opportunitätskosten - das ist der nunmehr auf $\bar{p}_{O}$ gesunkene Preis „an der Grenze“ - übersteigt. Das bedeutet eine Wohlstandserhöhung in Form des punktierten Dreiecks. Auch die vormals schon realisierte Importmenge $M_{O}^{0}$ wird nun zum geringeren Preis $\bar{p}_{O}$ verfügbar. Sofern diese Differenz auf den beseitigten Zoll zurückgeht, entsteht allerdings auch ein Verlust an Zolleinnahmen, so daß neben dem erwähnten D reieck als zusätzlicher Wohlstandseffekt lediglich die Einsparung der realen Transaktionskosten verbleibt, in der Abbildung 1 das horizontal schraffierte Rechteck.

2. H andelsumlenkung: Im Zuge der Verschiebung von $N_{R}^{0}$ (bzw. $N_{R}^{0 t}$ ) auf $N_{R}^{1}$ (bzw. $N_{R}^{1 t}$ ) verzichtet die heimische Wirtschaft auf Importgüter, deren Wert in den Augen der Nachfrager stets gleich $p_{R}^{0}$ ist. Das ist mehr als deren Opportunitätskosten, die dem ausländischen Produzentenpreis $\bar{p}_{R}$ plus den realen Transaktionskosten entsprechen. M.a.W., es entgeht der Ö konomie als $\mathrm{G}$ anzes Wohlstand im Ausmaß des punktierten Rechtecks, konkret in Form entgangener Zolleinnahmen.

3. Eine Verschiebung ähnlich jener für Importe aus Drittländern tritt auch für heimische G üter ein. Dies ist es wohl, was im Sinne des obigen Prinzips 2 als Bedrohung durch die Importe empfunden wird. Sofem aber diese Güter im Inland zu Preisen angeboten werden, die ihren Grenzkosten (Opportunitätskosten) entsprechen, können wir die Argumentation des vorigen Punktes nicht darauf übertragen. D.h., die Schrumpfung der heimischen Produktion ist nicht mit einem W ohlstandsverlust verbunden, es sei denn, die dadurch freigesetzten Inputs (etwa 
Arbeit) werden nicht anderswo wieder verwendet, wo sie einen ihren jeweiligen Preisen entsprechenden Wert schaffen.

Die Abbildung 1 betrachtet die Importseite, und sie suggeriert - ganz im Gegenteil weit verbreiteter Denkmuster - ein Potential für positive Wohlstandseffekte durch eine Zunahme der Importe aus den neuen Mitgliedsländem. Dies bedeutet freilich nicht, daß die Exportseite völlig belanglos ist. Eine zur Abbildung 1 spiegelbildliche Betrachtung der Exporte in die neuen Mitgliedsländer führt unmittelbar zur Identifikation von analogen Vorteilen fur die neuen Mitdiedsländer, denn unsere Exporte sind ja deren Importe. Es ist also demnach unsere Öffnung gegenüber den neuen Mitgliedsländem, die für uns vorteilhaft ist, und nicht die von den MOEL „gewährte" Liberalisierung.

Positive Effekte auf der E x portseite für bestehende Mitgliedsländer sind gleichwohl möglich, aber wiederum verleiten fehlgeleitete Prinzipien zu voreiligen Schlüssen Jede Zunahme der Exporte in neue Mitgliedsländer ist für die Ökonomie als ganzes mit Opportunitätskosten verbunden, und die entscheidende Frage ist, ob die dafür erzielten Exporterlöse diese Kosten übersteigen, ihnen entsprechen, oder gar danunter liegen. Letzteres ist z.B. dann der Fall, wenn die Exporte aus subventionierter Produktion stammen, und erst recht dann, wenn die Exporte als solche subventioniert werden. Dies mag in der Exportindustrie sehr erwünscht sein, aber gleichwohl bringt jede auf solche Weise zustande kommende Exportexpansion für die Ökonomie insgesamt eine Wohlstandseinbuße. Für Ö konomen ist dies trivial, aber in die Denkmuster wirtschaftspolitischer Diskussion hat es noch bedauerlich wenig Eingang gefunden.10 Positiv wirkt eine Exportzunahme hingegen dann, wenn damit eine Preissteigerung verbunden ist. Dies tritt z.B. dann ein, wenn perfekte Konkurrenz die Preise auf die Grenzkosten drückt, und wenn die Grenzkosten mit zunehmender Produktion ansteigen. Dann entsteht - unter ansonsten gleichbleibenden Bedingungen - eine wohlstandsmehrende Verbesserung der Terms-oftrade. Hier muß allerdings noch angemerkt werden, daß in spiegelbildlicher Weise auf der Importseite

10 Diese Bemerkungen unterstellen wettbewerbliche Gütermärkte. Die neue Theorie der Handelspolitik betont demgegenüber die Möglichkeit wohlstandssteigernder Exportsubventionen für heimische Firmen, die in oligopolistischem Wettbewerb mit ausländischen Firmen stehen. Hierzu ist Zweierlei zu sagen. Zum einen muß man unterscheiden zwischen der Frage, ob die E infiuhrung von Exportsubventionen bei gegebenen Handelsbamieren eine Wohlstandssteigerung beinhaltet, und der Frage, ob eine durch Handelsliberalisierung hervorgerufene Exportsteigerung in einer Welt bestehender Export- oder Produktionssubventionen wohlstandssteigernd ist. Die Theorie der strategischen Handelspolitik thematisiert die erste, unser Interesse gilt hier aber der zweiten Frage. Zum anderen ist wohl nicht von vornherein ausgeschlossen, daß die Beseitigung von realen Handelskosten in ihrer Wirkung auch eine strategische Komponente aufweisen, aber davon sind hier alle EULänder auf gleiche Weise betroffen, so daß eine Rentenumlenkung auf Firmen einzelner EU-Länder sehr fraglich erscheint. 
eine Verschlechterung der Terms-of-trade eintreten kann. In der Abbildung 1 wurde dies durch die Annahme konstanter Grenzkosten ausgeschlossen.

\section{Quantifizienung}

Kann man die eben beschriebenen Effekte im Kontext der O sterweiterung beziffern? Idealerweise müßte man zu diesem Zweck in der Lage sein, die Mengen- und Preiseffekte der Erweiterung im O stWest Handel abzuschätzen. Dies wiederum erfordert die Kenntnis und modellhafte Abbildung der in Abbildung 1 schematisch dargestellten Nachfrage- und Angebotsbedingungen, und zwar auf einer Vielzahl von Märkten sowohl auf der Export-, als auch auf der Importseite. Was tun, wenn man über ein solches Modell nicht verfügt? Interessante Anhaltspunkte lassen sich über eine empirische Ermittlung der in Abbildung 1 erscheinenden Wohlstandsrechtecke gewinnen. Es ist zu erwarten, daß diese Flächen von Gut zu Gut und Land zu Land durchaus unterschiedlich groß sind, je nach Zusammenspiel der Güterstruktur der Importe aus den neuen Mitgliedsländern mit dem EU-Außenzoll und den erwähnten „technischen“ Barrieren. In internationalen Vergleich scheint es naheliegend, diese Flächen zum jeweiligen BIP in Beziehung zu setzen.1.1

Die ersten beiden Spalten der Tabelle 2 präsentieren derartige Flächenmaße für die bestehenden EU-Länder, und zwar für den Beitritt der ersten 5 bzw. aller 10 oben erwähnten MOEL. Hinter diesen Zahlen verbergen sich Berechnungen einzelner Flächen für mehr als 5000 verschiedene Güter, die für jedes Land zu einem G esamtmaß aggregiert wurden. $M_{O 1}$ steht für das Zolleinnahmen-Rechteck und $M_{\mathrm{O} 2}$ für die Fläche der realen Handelskosten in der linken Hälfte der Abbildung 1. Nachdem die Zolleinnahmen im Rahmen des EU-Eigenmittelsystems der Gemeinschaft (und nicht dem Importland) zufließen, kann man $M_{O 1}$ als Maß dafür interpretieren, inwiefern ein bestimmtes Land im Rahmen der Osterweiterung zu Lasten anderer E U-Länder profitiert. Das Maß $M_{O 2}$ hingegen kann als Wohlstandsgewinn interpretiert werden, der sich aus der Ausdehnung des Gemeinsamen Marktes ergibt und nicht zu L asten anderer L änder geht. Eine andere Interpretation von $M_{O 1}$ nimmt Bezug auf das Wohlstandsdreieck in der Abbildung 1 und löst sich damit von der reinen Verteilungsperspektive. Dieses D reieck beträgt im Ausmaß die Hälfte des Maßes $M_{O 1}$, vorausgesetzt die Osterweiterung führt durchwegs zu einer Verdoppelung der Importe aus MOEL. Als empirische Aussage macht dies natürlich wenig Sinn, es dient hier lediglich als Referenzhypothese zur Interpretation des Maßes $M_{O 1}$. Zusammen genommen, können $M_{O 1}$ und $M_{O 2}$ als Indikator dafür angesehen werden, in welchen $\mathrm{G}$ rö-

\footnotetext{
${ }^{11}$ In Kohler (1999) werden derartige Flächenmaße auf der Basis eines Modells wohlstandsökonomisch interpretiert.
} 
ßenordnungen sich für die einzelnen EU-Länder die Handelsschaffungseffekte aus der O sterweiterung auf der Importseite bewegen. D ies erlaubt einerseits einen internationalen Vergleich, andererseits aber auch für jedes einzelne Land einen Vergleich mit dem Ausmaß der fiskalischen Belastung aus den Kosten der O sterweiterung (siehe unten). Allerdings ignoriert dies die Handelsumlenkungseffekte, in der rechten Hälfte der Abbildung 1 schematisch dargestellt als entgangene Zolleinnahmen. Diese Effekte werden durch die Substitutionsbeziehungen zwischen den Importgütern aus den MOEL und den Drittländem, sowie den heimischen Gütem bestimmt (siehe Panagariya, 1997). In Kohler (1999) werden diese Substitutionseffekte unter Rückgriff auf historisch beobachtete Handelsverschiebungen im Wege einer Korrektur der Maße $M_{O 1}$ und $M_{O 2}$ berücksichtigt. Die mit $\tilde{M}_{O}$ überschriebene Spalte der Tabelle 2 beinhaltet die Summe der beiden derart korrigierten Maße.

Die ersten sechs Spalten der Tabelle 2 nehmen eine die gesamte Wirtschatt betreffende Wohlstandsperspektive ein. In der Betonung von Vorteilen aus Importzuwächsen steht diese Perspektive, wie schon mehrfach betont, in einem gewissen Widerspruch zur Orthodoxie wirtschaftspolitischen Denkens. Die siebte Spalte stellt dem eine auf orthodoxe Weise exportbezogene Perspektive entgegen, wenn man so will - polit-ökonomisch formuliert - die Sicht der Produzenteninteressen in der E x portindustrie. Wie oben erwähnt, sind die positiven Exporteffekte ihrer Natur nach Terms-of-trade Verbesserungen im Export in die MOEL. Solche sind um so eher zu erwarten, je größer die Barrieren, mit de nen die EU-Exporte in den MOEL zunächst konfrontiert sind, und die im Zuge der Osterweiterung verschwinden werden. Dieser Überlegung folgend, kann man für jedes EU-Land ein gewichtetes Mittel aus den güterspezifischen Handelsbarrieren der MOEL errechnen, und zwar unter Verwendung des Anteils der von diesen Barrieren betroffenen Exporte am Inlandsprodukt des EU-Landes als $\mathrm{Ge}$ wicht. Die Ergebnisse derartiger Berechnungen in der mit $X_{O}$ überschriebenen Spalte wiedergegeben. Aus Gründen der Datenverfügbarkeit beschränkt sich dieses Maß allerdings auf die Gruppe der MOEL1.

\section{Der N ettoeffekt der O sterweiterung im internationalen Vergleich}

Die Tabelle 2 vermittelt in zweifacher Hinsicht ein unvollständiges Bild der Osterweiterung. Zum einen sind die fiskalischen Belastungen der einzelnen Länder noch nicht berücksichtigt, die sich aus den Kosten der 0 sterweiterung ergeben. Zum anderen beschränken sich die dort präsentierten Maße auf die traditionellen Handelseffekte. Sie lassen damit eine Reihe von weniger traditionellen Auswirkungen der Integration noch außer Acht, die vor allem mit Größenvorteilen, unvollständiger Konkurrenz, und Wachstum zu tun haben. Ich komme gleich darauf zurück. 


\section{Die Kosten der Erweiterung}

Nach dem in Berlin verabschiedeten Finanzrahmen der EU für die Periode 2000-2006 ergibt sich für die 15 bestehenden Mitgliedsländer aus der ersten Runde der Erweiterung ein Finanzierungsbedarf von etwas über 10 Mia. Euro, gemessen an den für 2006 geschätzten Zahlungen aus den verschiedenen Titeln der EU-Ausgabenpolitik und dem geschätzten Eigenmittelaufkommen der 5 neuen Mitglieder, jeweils zu Preisen von 1999. In Prozent des EU15-Sozialprodukts ausgedrückt, resultiert eine Belastung im Ausmaß von 0,113 Prozent; siehe European Council (1999) und European Commission (1999). Vergleicht man dies mit Kostenschätzungen auf der G rundlage von modellhaften Abbildungen der EU-Ausgabenpolitik, so ergeben sich geringfügig veränderte, meist etwas größere Belastungen. Baldwin et al. (1997) kommen unter Verwendung eines auf die Abstimmungsmacht im Europäischen Rat konzentrierten Modells für eine erste Erweiterungsrunde auf Kosten im Ausmaß von 0,11 und für eine weitere Runde auf 0,178 Prozent. ${ }^{1 / 2}$ Alternativ dazu kann man die beiden großen Ausgabenkategorien der Union, die Gemeinsame Agrarpolitik und die Europäische Strukturpolitik, mit Hilfe eines ökonometrischen Modells abbilden, das die relevanten ökonomischen Merkmale der Länder erfaßt. Wendet man ein von Breuss (1995) geschätztes Modell dieser Art an, so ergibt sich für die erste Erweiterungsrunde eine Belastung von 0,184 Prozent, und für beide Runden zusammen eine Belastung von 0,370 Prozent. ${ }^{13}$ Mit Hilfe dieses Modells lassen sich darüber hinaus die aus alternativen Finanzierungsszenarien resultierenden länderspezifischen Belastungen berechnen, die den bislang betonten Liberalisierungseffekten gegenübergestellt werden müssen. Die EU hat dabei im wesentlichen drei Optionen: a) eine Erhöhung der Beiträge, b) eine Reduktion der Agrarfonds, und c) eine Rückführung der Europäischen Strukturfonds.

Es ist klar, daß diese Optionen sehr unterschiedliche Verteilungen der Finanzierungslast auf die einzelnen Unionsländer bedeuten. D ie Tabelle 3 stellt die Unterschiede auf stilisierte Weise dar. Die erste

12 Die erste Erweitenung umfaßt bei diesen Berechnungen anstelle von Estland noch die Slowakei. Die obigen Zahlen basieren auf einer Messung der Abstimmungsmacht mit Hilfe des sogenannten Shapley-Shubik Index, der auf die Anzahl der Koalitionen abstellt, bei denen einem Land aufgrund seines Stimmengewichts eine entscheidende Bedeutung zukommt. Verwendet man statt dessen einfach die Stimmen pro Kopf der Bevölkerung, so ergibt sich für die erste Runde eine Belastung von 0,2 Prozent. Für die zweite Runde weisen Baldwin et al. keine alternativen Schätzwerte aus.

${ }^{13}$ Diese Zahlen sind zu interpretieren als jenes Budgetdefizit, das die erweiterte EU dann realisieren würde, wenn die bestehenden Politiken unverändert auf die neuen Mitgliedsländer angewandt würden. Nachdem die Kommission zu einem jährlichen Budgetausgleich verpflichtetist, muß die Budgetpolitik zur Vermeidung dieses D efizits verändert werden. Dafür wurden hier altemativ die im Text erwähnten Szenarien unterstellt, wobei die Veränderungen jeweils auf der Basis des ökonometrischen Modells und unter Einschluß der neuen Mitglieder berechnet wurden; siehe Keuschnigg \& Kohler (1999). 
Spalte zeigt für die EU-Länder die Beitragssätze des Jahres 1997, für die Beitrittsländer wird die Eigenmittelobergrenze von 1,28 Prozent angesetzt. In Verbindung mit den durch das ökonometrische Modell repräsentierten Ausgabenpolitiken der EU ergeben sich für die erweiterte EU die eben als Kosten der Erweiterung apostrophierten Budgetdefizite, in der Tabelle am unteren Ende wiedergegeben. Nun kann man sich fragen, wie die Beitragssätze angepaßt werden müßten, um bei konstanten Ausgaben einen Budgetausgleich zu erreichen. Unterstellt man als „Gerechtigkeitsnorm“ eine proportionale Anpassung für alle Länder, so ergeben sich die in Spalte 2 und 3 ausgewiesenen Beitragssätze. Die Spalten 4 bis 9 spiegeln auf analoge Weise das Resultat einer Anpassung der Agrarfonds bzw. Strukturfonds wider. Im Hinblick auf die doch sehr unterschiedlichen Belastungsmuster erhebt sich natürlich die Frage, welches die wahrscheinlichste Finanzierungsstrategie sein wird. Ein Anhaltspunkt ergibt sich aus dem Berliner Gipfel, wo im Vergleich zu bisherigen Vorschlägen die größte Anpassung im Bereich der Strukturfonds anvisiert wird. Zwar reichen die dort vorgesehenen Kürzungen zur Finanzierung der gesamten Kosten nicht aus, aber ein gewisses Schwergewicht in diesem Bereich wird gleichwohl sichtbar (siehe Keuschnigg \& Kohler, 1999). Vergleicht man die fiskalischen Belastungen der Tabelle 3 mit den Wohlstandseffekten der Tabelle 2, so erkennt man Zweierlei. Zum einen eine ausgeprägte Asymmetrie: Die Länder, welche durch die Finanzierungslasten relativ stark betroffen sind, sind nicht zugleich jene, die aus der Liberalisierung die größten Vorteile ziehen. Zum anderen zeigt sich, daß die Größenordnung der Wohlstandseffekte insgesamt deutlich unter den Finanzierungslasten liegen.

\section{Die modeme Theorie}

Müssen wir daraus schließen, daß die O sterweiterung für die bestehenden Mitgliedsländer per Saldo von Nachteil ist? Dieser Schluß wäre voreilig. Die bislang betonten Wohlstandseffekte sind nämlich insofern unvollständig, als sie eine Reihe von Wirkungskanälen unbeachtet lassen, die in der modernen Integrationstheorie besonders betont werden. Man kann sie insofern als "traditionelle" Effekte bezeichnen, die einer Ergänzung im Lichte der modernen Theorie bedürfen. Es geht dabei um unvollständiger Konkurrenz, um Größenvorteile in der Produktion, und um Wachstumseffekte - alles Phänomene, die in der traditionellen Theorie stiefmütterlich behandelt wurden, und erst in den letzten 15 Jahren eine auch für die empirische Forschung tragfähige Verankerung in der Theorie der Integration gefunden haben; siehe Baldwin \& Venables (1995). Der für eine empirische Quantifizierung erforderliche Modellierungsaufwand würde indessen den Rahmen dieser Arbeit sprengen. Die Effekte sind nämlich weitgehend indirekter Art, und sie entziehen sich einer empirischen Erfassung über eine Betrachtung von Handelsbarrieren nach dem Muster der Abbildung 1 und der Tabelle 2. Indirekt heilst hier aber mitnichten von geninger Bedeutung. In der Tat suggeriert die modeme Theorie, daß diese Effekte in ihrer Bedeutung die traditionellen Effekte sogar dominieren. 
Es fehlt hier leider der Platz für eine ins Detail gehende Erörterung. Wir müssen uns mit einigen ganz kurzen Andeutungen, sowie mit einer Anleihe bei an anderer Stelle ausführlicher präsentierten Ergebnissen begnügen. Der wohl wichtigste Aspekt betrifft die W achstumseffekte. Rational agierende Firmen wählen jene Ausdehnung des Kapitalstocks, bei der eine zusätzliche Einheit Kapital pro Periode genau so viel Kosten verursacht, wie sie zusätzliche Wertschöpfung generiert. Im langfristigen Gleichgewicht bestehen die Kosten aus $(i+\delta) P_{K}$, wobei $i$ der Kapitalmarktzinssatz, $\delta$ die Abschreibungsrate, und $P_{K}$ der Anschaffungspreis für eine Einheit Kapital darstellt. Man spricht hier auch von den langfristigen Nutzungskosten des Kapitals. Die zusätzliche Wertschöpfung ergibt sich aus dem durch die zusätzliche Kapitaleinheit möglich gewordenen zusätzlichen Outputwert, d.h. der

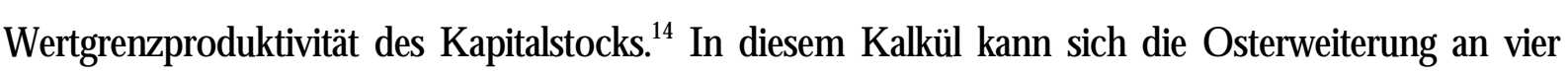
verschiedenen Stellen niederschlagen. a) Die erhöhte Exportnachfrage kann zu einer O utputpreiserhöhung, und damit zu einem Anstieg der Wertgrenzproduktivität des Kapitals führen - für sich genommen ein Investitionsanreiz. b) Die verbilligten Importe können zu Kostenersparnissen im Bereich der Vorprodukte führen, was wiederum die Wertschöpfung und damit auch die Wertgrenzproduktivität des Kapitals erhöht. Daraus ergibt sich wie zuvor ein Investitionsanreiz. c) Die verbilligten Importe können den Anschaffungspreis für Kapitalgüter senken, woraus sich geringere Nutzungskosten des Kapitalstocks und ein weiterer Investitionsanreiz ergeben. d) Schließlich kann sich aufgrund der Osterweiterung eine Veränderung des Kapitalmarktzinssatzes ergeben. Eine Erhöhung würde die Nutzungskosten des Kapitals erhöhen und für sich genommen kontraktiv auf den Kapitalstock wirken. D as G egenteil gilt für eine Zinssenkung. Es wird gemeinhin argumentiert, daß die Transformation und Öffnung der MOEL aufgrund des großen Kapitalbedarfs mit einem Zinssteigerungseffekt verbunden ist. Ob allerdings die Osterweiterung der EU für sich genommen eine zusätzliche Steigerung verursachen wird, ist sehr fraglich. Bei konstantem Kapitalmarktzins ist aus den Effekten a) bis c) ein expansiver Effekt auf den Kapitalstock in den bestehenden EU-Ländern zu erwarten. Dabei handelt es sich um einen einmaligen $N$ iveauefelkt bei konstanter (exogener) langfristiger Wachstumsrate. Prinzipiell ist sogar denkbar, daß ein Integrationsschritt wie die O sterweiterung auch die langfristige $W$ achstumsrate der bestehenden Mitglieder verändert. Die Theorie des endogenen Wachstums benennt eine Reihe von Umständen, unter denen dies der Fall sein kann, aber es ist im Falle der O sterweiterung außerordentlich schwierig, von der prinzipiellen Möglichkeit zu empirisch einigermaßen abgesicherten Effekten zu gelangen. Im folgenden wird deshalb eine exogene, durch die O sterweiterung nicht beeinflußte Wachstumsrate angenommen.

\footnotetext{
${ }^{14}$ Dieses Argument unterstellt einen abnehmenden Verlauf der Grenzproduktivität des Kapitalstocks.
} 
Angenommen also, die Osterweiterung hat einen einmaligen Niveaueffekt auf den Kapitalstock in bestehenden Mitgliedsländem. Wie ist dieser Effekt zu vergleichen mit den Wohlstandseffekten der obigen Abbildung 1? Nicht selten wird der Eindruck erweckt, daß Wachstumseffekte 1:1 auch Wohlstandseffekte darstellen. Dies ist falsch! Ähnlich wie in der Abbildung 1 die zusätzlichen Importe aus MOEL für die Ökonomie zusätzliche Kosten verursachen, und zwar im Ausmaß von $\bar{p}_{O} \times\left(M_{O}^{1}-M_{O}^{0}\right)$, so kommt auch der zusätzliche Kapitalstock nicht gratis, sondern ist mit Kosten im Ausmaß von $(i+\delta) P_{K} \times\left(K^{1}-K^{0}\right)$ verbunden. Der Wohlstandseffekt beschränkt sich auf das Ausmaß, in dem der zusätzliche Kapitalstock $K^{1}-K^{0}$ eine Wertschöpfung generiert, die diese Kosten übersteigt (analog zum punktierten Wohlstandsdreieck in der Abbildung 1).

In einer Welt unvollständiger Konk urrenz liegen die Preise typischerweise über den G renzkosten. Ähnlich wie eine Importerhöhung dann mit einer Wohlstandszunahme verbunden ist, wenn der Inlandspreis - bedingt durch Importbarrieren - über dem Preis „an der Grenze“ liegt (siehe Abbildung 1), so ist auch eine inländische Produktionsausdehnung dann unmittelbar von Vorteil, wenn der Preis (und damit auch die marginale Zahlungsbereitschaft der Nachfrager) für dieses Gut die G renzkosten der Erzeugung (und damit auch die O pportunitätskosten für die Ö konomie) übersteigt. M.a.W., in solchen Fällen ist ein Wachstumseffekt von der eben beschriebenen Art mit einem zusätzlichen Wohlstandseffekt verbunden. Unvollständige Konkurrenz ist häufig auch mit der Existenz von G rößenvorteilen, etwa in Form von firmenspezifischen Fixkosten, verbunden. In diesem Falle ist natürlich von Belang, ob das Wachstum durch neue Firmengründungen erfolgt, verbunden mit neuen Fixkosten, oder durch Firmenwachstum, verbunden mit Kostendegression. In einer durch Produktdifferenzierung charakterisierten Wirtschaft tut sich hier ein Spannungsfeld auf zwischen Produktvielfalt auf der einen, und Fixkostendegression auf der anderen Seite. Ein weiterer wohlstandsrelevanter Effekt des Wachstums kann daher danin bestehen, daß der Trade-off zwischen Produktvielfalt und Größenvorteil gewissermaßen „nach außen verschoben“ wird, daß mithin von beidem zugleich mehr erreicht werden kann.

Wie können nun diese „modernen“ Effekte beziffert werden? Was man streng genommen benötigt, ist ein entsprechend reichhaltig spezifiziertes und empirisch implementiertes Modell, das eine direkte Abschätzung der eben angedeuteten Effekte erlaubt. Ein Modell dieser Art, das auf vergleichbare Weise alle EU-Länder zugleich abdeckt, steht indes nicht zur Verfügung, und es wäre wohl auch vermessen, ein solches überhaupt anzustreben. Was jedoch möglich scheint, das ist eine Übertragung von Ergebnissen, die mit Hilfe eines solchen Modells für den österreichischen Fall berechnet wurden (siehe Keuschnigg \& Kohler, 1999), auf die anderen EU-Länder. In Kohler (1999) habe ich eine Methode der Übertragung vorgestellt. Sie basiert im wesentlichen auf der Annahme, daß die in Tabelle 2 präsen- 
tierten "traditionellen" Effekte auch Aufschluß geben können über das Ausmaß, in dem ein Land durch "moderne“ Effekte von der Osterweiterung profitiert. So läßt z.B. der Index $\tilde{M}_{O}$ darauf schließen, um wieviel weniger Dänemark durch die importseitigen Wohlstandseffekte von der O sterweiterung profitiert als Ö sterreich. Für die Erweiterung auf MOEL1 beträgt der Effekt für Dänemark z.B. nur 35,2 Prozent $(=100 \times 0,0634 / 0,18)$ des Effekts für Österreich. Unterstellt man nun, daß diese Relation auch für die durch $\tilde{M}_{O}$ noch nicht abgedeckten „modernen“ Effekte gilt, und steht im Falle Ö sterreichs ein empirischer Schätzwert für den Gesamteffekt - einschließlich der "modemen“ Effekte, aber ohne Berücksichtigung der fiskalischen Belastung - zur Verfügung, dann läßt sich daraus auf indirekte Weise auf den G esamteffekt für D änemark schließen.

Was den Gesamteffekt für Österreich anlangt, so greife ich auf ein Simulationsergebnis zurück, das mit Hilfe eines allgemeinen Gleichgewichtsmodells erzielt wurde. Dieses beinhaltet die eben angedeuteten, „modernen“ Aspekte der Integrationstheorie, und es wurde im Wege der Kalibrierung für den Spezialfall Ö sterreich empirisch implementiert. ${ }^{1.5}$ Die Abbildung 2 zeigt das Ergebnis der Übertragung auf der Basis eines ungewichteten arithmetischen Mittels mehrener Indizes betreffend die „traditionellen" Effekte. Diese erfassen neben den Import- und Exportaspekten der Tabelle 2 auch Terms-oftrade Effekte; siehe dazu Kohler (1999). Die Länder sind nach der Größe dieses Effektes geordnet. Die Linie INTEGR zeigt den nach der eben skizzierten Methode geschätzten Gesamtwohlstandseffekt aus der Gütermarktintegration, und zwar beschränkt auf die MOE1-Länder, und in Prozent des jeweiligen BIP. Dem werden in Form von Balken die fiskalischen Belastungen aus den Kosten der Erweiterung gegenübergestellt, und zwar für die drei oben erwähnten Szenarien und ebenfalls in Prozent des BIP. BEITR steht für eine proportionale Anhebung der Beitragszahlungen, GAP für eine proportionale Reduktion der Rückflüsse aus der Gemeinsamen Agrarpolitik, und ESF für eine proportionale Rückführung der Mittel aus den Europäischen Strukturfonds. Es zeigt sich, daß drei Länder durch die Gütermarktintegration stärker von der O sterweiterung profitieren, als sie durch den Nettomittelabluß an die neuen Mitglieder zur Kasse gebeten werden, und zwar unabhängig von der auf EUEbene gewählten Finanzierungsstrategie. Diese „Gewinner" sind Deutschland, Finnland und Österreich. Auf der anderen Seite stehen vier Länder, die bei diesem Kalkül unabhängig von der Finanzierungsstrategie als „Verlierer" ausgewiesen werden; das sind Griechenland, Illand, Portugal, und Spa-

\footnotetext{
15 Es fehlt hier der Platz selbst für eine oberflächliche Beschreibung dieses Modells, aber die intendierte Botschaft ist zweifellos auch so verständlich. Überblicksartige Darstellungen des Modells sind enthalten in Keuschnigg \& Kohler (1996a, 1996b,1998, und 2000), eine ausfühnliche Präsentation der Ergebnisse und der Methode ist enthalten in Keuschnigg \& Kohler (1999). D iese letztere Arbeit ist verfügbar unter http:/ / www.economics.uni-linz.ac.at/ members/ kohler/ eustud.htm.
} 
nien. Für die Mehrheit der dazwischen liegenden Länder hängt der Nettoeffekt von der Art und Weise ab, wie die Kosten der Erweiterung finanziert werden. Nimmt man den Ratsbeschluß von Berlin als Bekenntnis zur Kürzung der Strukturfonds als primären Weg der Finanzierung, so treten vier weitere Länder zu den „Gewinnern“, nämlich Dänemark, Italien (knapp), die Niederlande und Schweden, während Belgien/ Luxemburg zu den „Verlierern“ tritt. Die beiden verbleibenden Länder Frankreich und Großsbritannien liegen in einer Zone der „Neutralität”.

\section{Was haben wir gelernt?}

Was kann man aus diesen Ergebnissen schließen? Die vielleicht wichtigste Botschaft ist schlicht und einfach die, daß die O sterweiterung für die bestehenden Mitgliedsländer nicht nur die allseits betonten fiskalischen Belastungen bringen wird, sondern auch die V orteile eines größeren Integrationsraumes, die zumindest annähernd auch bezifferbar sind, und somit den Budgeteffekten gegenübergestellt werden können. So gesehen steht zu hoffen, daß dieser Beitrag dazu verhilft, die Diskussion um die O sterweiterung auf eine etwas breitere und ausgewogenere Basis zu stellen. Allerdings muß abschließend noch eigens betont werden, daß die hier vorgestellten Berechnungen sich vorerst ausschließlich auf die Gütermarktintegration beschränken. Man mag einwenden, daß insbesondere die Vernachlässigung der Migration insofern eine gravierende Einschränkung darstellt, als die politische Diskussion sich sehr stark genau auf diesen Aspekt konzentriert. Daß in diesem Punkt eine Ergänzung der hier vorgenommenen Betrachtung not tut, sei zugestanden. Andererseits ist aber wohl zu erwarten, daß die Beitrittsverträge den vollen Binnenmarktstatus auf den Arbeitsmärkten erst nach gewissen Übergangsfristen vorsehen werden, so daß die erweiterungsbedingten Migrationsströme erst mit erheblicher Verzögerung eintreten werden und aus heutiger Sicht nur mit großer Unsicherheit abschätzbar sind. Ein weiterer Punkt be trifft die D irek tinvestitionen in neuen Mitgliedsländem, die hier ebenfalls außer Acht gelassen wurden. Ich habe in Kohler (1999) argumentiert, daß die Osterweiterung zwar durchaus zu einem zusätzlichen Investitionsstrom von bestehenden in die neuen Mitgliedsländer führen kann, daß deren Wohlstands-

wirkungen in den westlichen Ländern aber sehr beschränkt sein dürften, so daß eine Ergänzung der Betrachtung in diesem Punkt weniger zwingend erscheint.

Von welcher Bedeutung sind die hier präsentierten Ergebnisse für den anstehenden Prozeß der Entscheidungsfindung zur O sterweiterung? Die Europäische Union hat in Maastricht beschlossen, die Entscheidung über eine Aufnahme neuer Mitglieder - nach vorheriger Anhörung der Kommission und Zustimmung des Europäischen Parlaments - durch einen einstimmigen Beschluß des E uropäischen Rates zu treffen. Wäre der Rat ein an gesamteuropäischen Interessen orientiertes Gremium, so müßte wohl berücksichtigt werden, daß die oben jeweils in Prozent des nationalen BIPs ausgewiesenen Effekte sich 
auf sehr unterschiedlich große Länder beziehen, in der Abbildung 2 ergänzungsweise durch die Bevölkerungszahlen angedeutet. Ein solches Gremium ist der Rat indessen nicht; vielmehr sind dort die Interessen der einzelnen Mitgliedsländer vertreten. Insofern ist die hier eingenommene Perspektive zweifellos von großer Bedeutung. Gälte nun das andere Extrem, würde also im Rat allein nach nationalen $W$ ohlstandskriterien entschieden, so käme es nach den hier vorgelegten Berechnungen nicht zur Osterweiterung, weil einzelne Länder dadurch verlieren. Zieht man die in Abbildung 2 aufgelistete Stimmenverteilung in Betracht, so erkennt man, daß die Erweiterung wohl auch dann nicht zustande käme, wenn sie per Mehrheitsentscheidung erfolgen könnte.

Nun ist aber nicht zu erwarten, daß dieses Kriterium im Rat wirklich auf diese einfache Weise dominieren wird. Zum einen habe ich oben schon darauf hingewiesen, daß die nationalen Regierungen in hohem Maße unter dem D ruck von partikulären Interessen (im Unterschied zum Gesamtwohlstand) stehen. Insofern lassen die hier präsentierten Ergebnisse - von den schon mehrfach betonten methodischen Einschränkungen einmal ganz abgesehen - nur sehr bedingt Rückschlüsse auf das Verhalten der Ratsmitglieder zu. Immerhin kann dazu abschließend noch ergänzt werden, daß positive Wohlstandseffekte der hier präsentierten Art bei Existenz von damit zusammenhängenden Verteilungskonflikten so interpretiert werden können, daß sie innerhalb eines Landes eine Kompensation der "Verlierer" ermöglichen. Zum anderen ist aus dem Europäischen Rat, wie auch aus anderen Beispielen bekannt, daß sukzessive einstimmig zu fällende Entscheidungen nicht unabhängig voneinander gesehen werden dürfen. Vielmehr wird typischerweise die Zustimmung zu einer für manche Mitglieder nachteiligen Maßnahme seitens der daran interessierten Mitglieder „enkauft" durch die Zustimmung zu anderen Maßnahmen mit einer gegenteiligen Interessenlage. Es wird im allgemeinen zu erwarten sein, daß die Aufnahme neuer Mitglieder die in wiederholten Abstimmungen gegebene „politische Macht" der bestehenden Mitglieder nicht unverändert läßt. Für den Fall der EU hat Wildgrén (1994) im Wege einer formalen Analyse der Abstimmungsmechanismen gezeigt, daß die A ufnahme neuer Länder tendenziell dazu führt, daß große Mitglieder an politischer Macht verlieren. Casella (1996) weist darauf hin, daß man diese Frage auch anders stellen kann: Wenn aufgrund des Einstimmigkeitsprinzips manchen Ländem die Zustimmung zu einer Erweiterung „abgekauft“ werden muß, weil sie dadurch eigentlich verlieren, dann werden diese Länder im Rahmen der G emeinschaft zumindest temporär an „politischer Macht" gewinnen. Dies verleitet dazu, zur Abbildung 2 zurückzukehren, und ... 


\section{Literatur}

Bagwell, Kyle and Robert W. Staiger, „An economic theory of GATT," The A merican E conomic Review 89 (1999):215-248.

Bagwell, Kyle and Robert W. Staiger, „Will preferential agreements undermine the multilateral trading system?" The E onomic Journal 108 (1998):1162-1182.

Baldwin Richard E. and Anthony J. Venables, „Regional economic integration,” in G ene M. Grossman and Kenneth Rogoff (eds.), H andbook of International E conomics, Vol. III, Amsterdam: Elsevier Science B.V. (1995):1597-1644.

Baldwin, Richard E., „A domino theory of regionalism,” in Richard E. Baldwin, Pertti Haaparanta and Jaakko Kiander (eds.), Expanding M embership of the E U, Cambridge: Cambridge University Press (1996): 25-48.

Baldwin, Richard E., Joseph F. Francois and Richard Portes, „The Costs and Benefits of Eastern Enlargement: The Impact on the EU and Central Europe," E conomic Policy (1997):127-176.

Bhagwati, Jagdish N. and Arvind Panagariya, „Preferential trading areas and multilateralism strangers, friends or foes," in Jagdish N. Bhagwati and Arvind Panagariya (eds.), The E conomics of Preferential Trade A greements, Washington D .C.: American Enterprise Institute, (1996):1-78.

Bhagwati, Jagdish N., „Regionalism and multilateralism: An overview,” in Jaime de Melo and Arvind Panagariya (eds.), N ew D imensions in Regional Integration, Cambridge: Cambridge University Press (1993): 22-51.

Bhagwati, Jagdish N., D avid Greenaway and Arvind Panagariya, „Trading preferentially: Theory and policy," The E conomic Journal 109 (1998):1128-1148.

Brenton, Paul, Trade and Investment in E urope: The Impact of the N ext E nlargement, Brussels: Centre for European Policy Studies (1999).

Breuss, Fritz, Costs and Benefits of E U's E astern E nlargement, Helsinki: Yrjö Jahnsson European Integration Lectures 5 (1995).

Breuss, Fritz and Fritz Schebeck, „0 stöffnung und 0 sterweiterung der E U," WIFO Monatsberichte 2/ 1996:139-151.

Breuss, Fritz and Fritz Schebeck, „Costs and Benefits of E U's E astern for A ustria,” Austrian Economic Quarterly 4/ 1999:43-54.

Casella, Alessandra, „Large countries, small countries and the enlargement of trade blocs,” E uropean E conomic Review 40 (1996):389-415.

D eardorff, Alan V. and Robert M. Stern, „Multilateral trade negotiations and preferential trading arrangements," in Alan V. Deardorff and Robert M. Stern (eds.), A nalytical and N egotiationg issues in the G lobal Trading System, Ann Arbor: University of Michigan Press (1994):53-85.

Ethier, Wilfred J., „The new regionalism,” The E conomic Journal 108 (1998):1149-1161.

European Commission, A genda 2000, European Commission, Luxembourg (1997).

European Commission, Financing the E uropean Union. Commission Report on the 0 peration of the $0 \mathrm{wn}$ Resources System, Brussels: European Commission (1998).

European Commission, Interinstitutional A greement Between the E uropean Parliament, The Council and The E uropean C ommission of 6 May 1999 on Budgetary D iscipline and Improvement of the Budgetary Procedure, Brussels: European Commission (1999). 
European Council, Presidency Conclusion - Berlin E uropean Council, 24 and 25 March 1999, Berlin: European Council (1999).

Finger, Michael J., Merlinda D. Ingco and Ulrich Reincke, The U ruguay Round: Statistics on Tariff C oncessions G iven and Received, Washington D .C.: The World Bank (1996).

Keuschnigg, Christian and Wilhelm Kohler, „Commencial policy and dynamic adjustment under monopolistic competition," Journal of International E conomics 40 (1996a):373-409.

Keuschnigg, Christian and Wilhelm Kohler, „Austria in the European Union: Dynamic Gains from Integration and D istributional Implications,” E conomic Policy 22 (1996b):155-211.

Keuschnigg, Christian and Wilhelm Kohler, E astern E nlargement of the E U : H ow M uch is it W orth for A ustria, London: CEPR Working Paper NO. 1786 (1998).

Keuschnigg, Christian and Wilhelm Kohler, E astern E nlargement of the E U : E conomic C osts and Bene fits for the E U Present M ember States, Brussels: Study XIX/ B1/ 9801 for the European Commission (1999).

Keuschnigg, Christian and Wilhelm Kohler, „Eastern enlargement of the EU: A general equilibrium perspective," in Glenn E. A. Harrison et al. (eds.), U sing D ynamic $G$ eneral E quilibrium M odels for Policy A nalysis. Amsterdam: North-Holland (2000).

Kohler, Wilhelm, W er gewinnt, wer verliert durch die 0 sterweiterung der E U , Arbeitspapier Nr. 9920, Johannes Kepler Universität Linz, Institut für Volkswirtschaftslehre (1999), Plenarvortrag bei der Jahrestagung 1999 des Vereins für Socialpolitik, 28.9.-1.10.1999, Mainz, erscheint im Tagungsband.

Krishna, Pravin, „Regionalism and multilateralism: A political economy approach,” Q uarterly Journal of E conomics 113 (1998):227-251.

Krugman, Paul R., „The move to free trade zones,” in Policy Implications of Trade and C urrency Z ones, Kansas City: Federal Reserve Bank of Kansas City (1991a):7-41.

Krugman, Paul R., „Is bilateralism bad?” in Elhanan Helpman and Assaf Razin (eds.), International Trade and Trade Policy, Cambridge, MA: MIT Press (1991b).

Krugman, Paul R., „Regionalism versus multilateralism: Analytical notes,” in Jaime de Melo and Arvind Panagariya (eds.), N ew D imensions in Regional Integration, Cambridge: Cambridge University Press (1993):58-79.

Leonard, D aniel, G uide to the E uropean Union, 6th ed., London: The E conomist in association with Profile Books Ltd. (1998).

Levy, Philip I., „A political-economic analysis of free-trade agreements,” The A merican E conomic Review 87 (1997):506-519.

Panagariya, Arvind, „The meade model of preferential trading: History, analytics, and policy implications," in Benjamin Cohen (ed.), International Trade and Finance: N ew Frontiers for Research, E ssays in H onor of Peter B. Kenen, New Y ork: Cambridge University Press (1997):57-88.

Summers, Lawrence, „Regionalism and the world trading system,” in Policy Implications of Trade and C urrency Z ones, Kansas City: Federal Reserve Bank of Kansas City (1991):295-301.

Wildgrén, Mika, „Voting Power in the EU D ecision Making and the Consequences of Two Different Enlargements," E uropean E conomic R eview 38 (1994):1153-1170. 
Abbildung 1

Wohlstandseffekte der Ostermeitenung durch Handelsliberalisienung

Importe aus MOEL

E reale Transaktionskosten

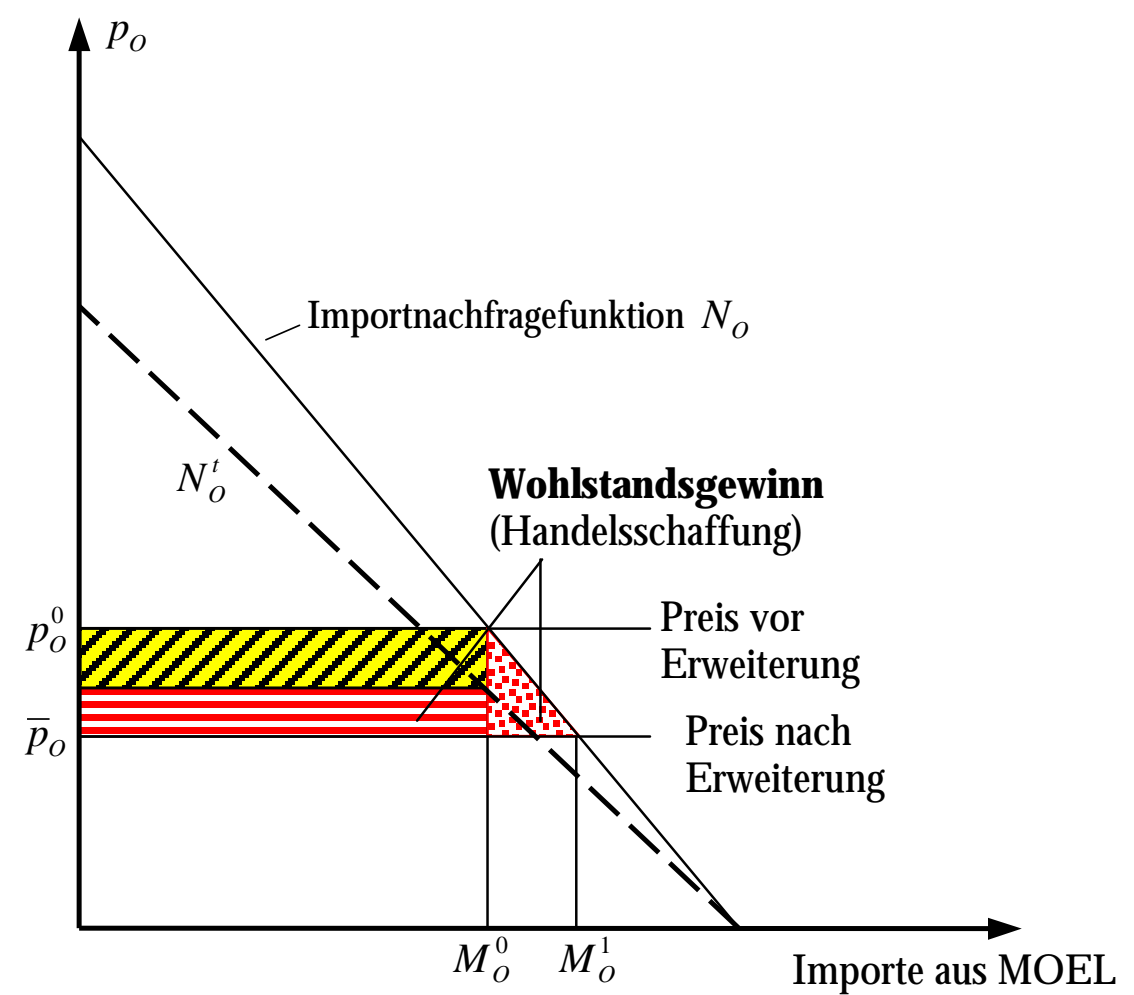

Importe aus Drittländem (,Rest der Welt")

8 Zolleinnahmen entgangene Zolleinnahmen

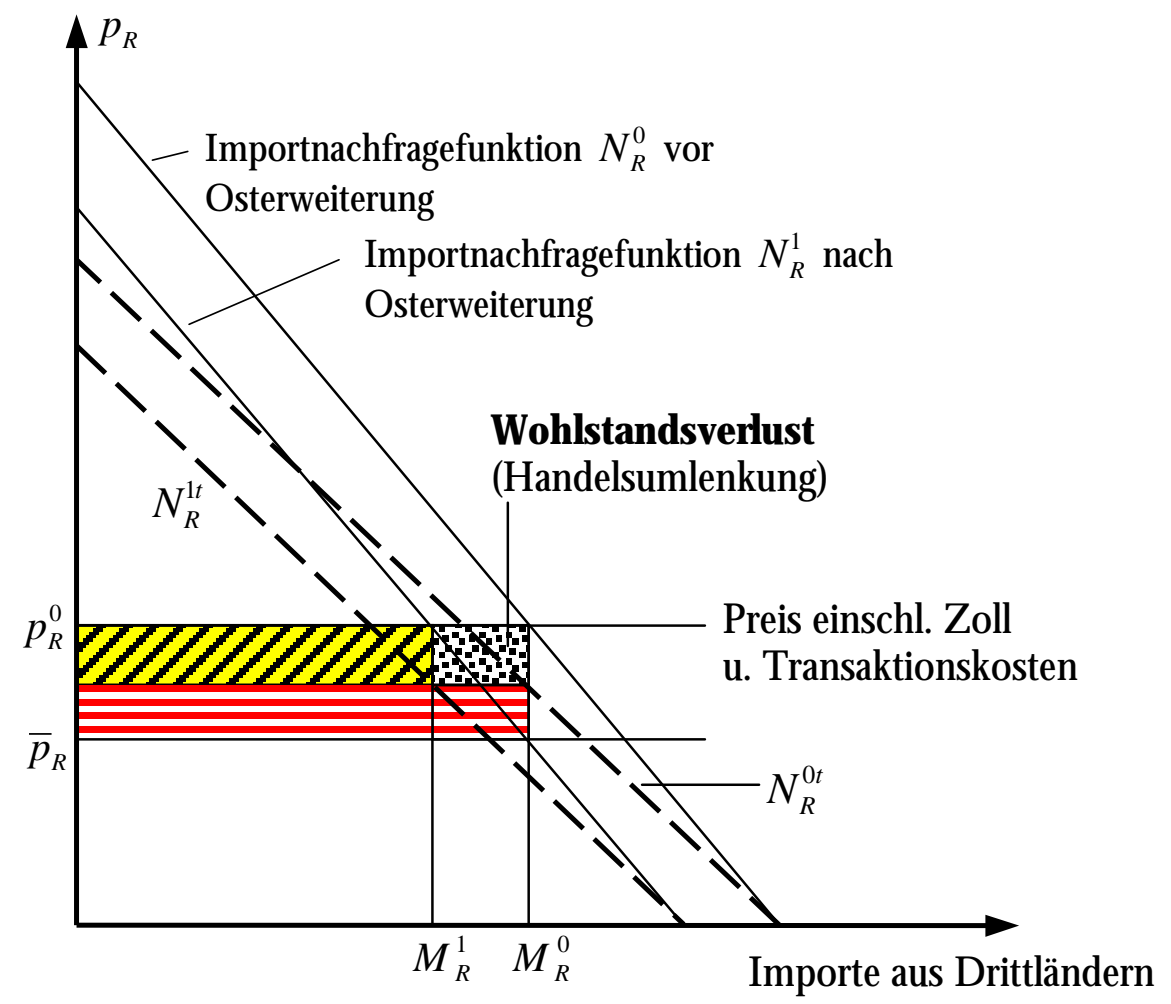


Tabelle 1

Exporte und Importe in die 10 MOE Länder

in $\%$ des BIP

\begin{tabular}{|l|cc|cc|}
\hline \hline \multicolumn{1}{|c|}{ Land } & $\begin{array}{c}\text { Exporte 1997 } \\
\text { in \% des BIP }\end{array}$ & $\begin{array}{c}\text { \%-Veränd. } \\
\text { 1993-1997 }\end{array}$ & $\begin{array}{c}\text { Importe 1997 } \\
\text { in \% des BIP }\end{array}$ & $\begin{array}{r}\text { \%-Veränd. } \\
\text { 1993-1997 }\end{array}$ \\
\hline Belgien / Lux. & 1,32 & 0,72 & 0,77 & 0,41 \\
\hline Dänemark & 1,09 & 0,43 & 0,81 & 0,24 \\
\hline D eutschland & 1,83 & 0,84 & 1,52 & 0,65 \\
\hline Finnland & 2,71 & 1,42 & 0,94 & 0,29 \\
\hline Frankreich & 0,51 & 0,27 & 0,32 & 0,13 \\
\hline Griechenland & 0,30 & $-0,06$ & 0,63 & 0,20 \\
\hline Großbritannien & 0,42 & 0,18 & 0,33 & 0,12 \\
\hline Irland & 0,33 & 0,24 & 0,16 & 0,07 \\
\hline Italien & 1,09 & 0,48 & 0,66 & 0,28 \\
\hline Niederlande & 1,03 & 0,25 & 0,79 & 0,18 \\
\hline Österreich & 3,98 & 1,62 & 2,71 & 1,17 \\
\hline Portugal & 0,20 & 0,15 & 0,17 & 0,08 \\
\hline Schweden & 1,45 & 0,82 & 1,05 & 0,55 \\
\hline Spanien & 0,39 & 0,24 & 0,26 & 0,15 \\
\hline EU 15 & $\mathbf{1 , 0 8}$ & $\mathbf{0 , 4 7}$ & $\mathbf{0 , 7 9}$ & $\mathbf{0 , 3 1}$ \\
\hline \hline
\end{tabular}

Q uelle: Breuss \& Schebeck (1999) sowie IMF International Financial Statistics.

Die 10 mittel -und osteuropäischen Länder (MOEL) sind: Bulgarien, Estland, Lettland, Litauen, Polen, Rumänien, Slowakei, Slowenien, Tschechien, Ungarn. 
Tabelle 2

Wohlstandseffekte der Gütermarktintegration im Zuge der Osterweitenung der EU in $\%$ des BIP

\begin{tabular}{|l||c|c|c|c|c|c|c|}
\hline \hline \multirow{2}{*}{\multicolumn{1}{|c||}{ LAND }} & \multicolumn{2}{c|}{$M_{O 1}$} & \multicolumn{2}{c|}{$M_{O 2}$} & \multicolumn{2}{c|}{$\tilde{M}_{O}$} & $X_{O}$ \\
\cline { 2 - 8 } & MOEL1 & MOEL1+2 & MOEL1 & MOEL1+2 & MOEL1 & MOEL1+2 & MOEL1 \\
\hline Belgien / Lux. & 0.0320 & 0.0455 & 0.0301 & 0.0393 & 0.0565 & 0.0777 & 0.1319 \\
\hline Dänemark & 0.0375 & 0.0502 & 0.0278 & 0.0348 & 0.0634 & 0.0807 & 0.0831 \\
\hline D eutschland & 0.0616 & 0.0776 & 0.0488 & 0.0593 & 0.1054 & 0.1293 & 0.1363 \\
\hline Finnland & 0.0359 & 0.0436 & 0.0344 & 0.0392 & 0.0694 & 0.0811 & 0.1917 \\
\hline Frankreich & 0.0168 & 0.0231 & 0.0123 & 0.0163 & 0.0280 & 0.0378 & 0.0475 \\
\hline Griechenland & 0.0269 & 0.0718 & 0.0142 & 0.0380 & 0.0384 & 0.0945 & 0.0249 \\
\hline Großbritannien & 0.0145 & 0.0194 & 0.0113 & 0.0152 & 0.0241 & 0.0323 & 0.0350 \\
\hline Irland & 0.0131 & 0.0152 & 0.0160 & 0.0187 & 0.0278 & 0.0322 & 0.0634 \\
\hline Italien & 0.0395 & 0.0595 & 0.0214 & 0.0334 & 0.0601 & 0.0871 & 0.0919 \\
\hline Niederlande & 0.0436 & 0.0623 & 0.0264 & 0.0391 & 0.0670 & 0.0976 & 0.0852 \\
\hline Ö sterreich & 0.1009 & 0.1238 & 0.0936 & 0.1127 & 0.1800 & 0.2193 & 0.2772 \\
\hline Portugal & 0.0084 & 0.0103 & 0.0046 & 0.0071 & 0.0120 & 0.0158 & 0.0134 \\
\hline Schweden & 0.0412 & 0.0501 & 0.0273 & 0.0385 & 0.0677 & 0.0862 & 0.0888 \\
\hline Spanien & 0.0137 & 0.0169 & 0.0075 & 0.0102 & 0.0199 & 0.0250 & 0.0306 \\
\hline EU15 & $\mathbf{0 . 0 3 7 4}$ & $\mathbf{0 . 0 4 9 9}$ & $\mathbf{0 . 0 2 7 8}$ & $\mathbf{0 . 0 3 6 2}$ & $\mathbf{0 . 0 6 4 3}$ & $\mathbf{0 . 0 8 4 1}$ & $\mathbf{0 . 0 8 9 7}$ \\
\hline \hline
\end{tabular}

Zur Interpretation der Indices $M_{O 1}, M_{O 2}, \tilde{M}_{O}$ und $X_{O}$ siehe Text.

MOEL1: Estland, Polen, Slowenien, Tschechien, Ungarn.

MOEL2: Bulgarien, Lettland, Litauen, Rumänien, Slowakei

Für die EU15 als Ganzes wurden eigene Berechnungen durchgeführt. D ie Zahlen sind also keine Länderdurchschnitte.

Q uelle: Berechnungen auf der Grundlage der ITCS-D atenbank der OECD . Diese umfaßt den Güterhandel nach mehr als 5000 Gütergruppen des Harmonisierten Systems, entsprechend disaggregiert nach Herkunfts und Bestimmungsländern. Für die Zölle wurde die O ECD-D atenbank „Indicators of Tariff and Non-tariff Trade Barriers" verwendet. Die Zahlen beziehen sich auf das Jahr 1997. Die Zölle für MO EL wurden entnommen aus Finger, Ingco \& Reincke (1996). Für weitere Details siehe Kohler (1999). Für die realen Handelskosten wurde ein Satz von 5 Prozent, bezogen auf den Transaktionswert unterstellt. D as bewegt sich am unteren Ende des "Literaturkonsenses“; siehe auch Baldwin et al. (1997). 
Tabelle 3

Beitragszahlungen und Rückflüsse für alte und neue EU Mitgliedsländer

in $\%$ des BIP

\begin{tabular}{|c|c|c|c|c|c|c|c|c|c|}
\hline \multirow[t]{2}{*}{ Land } & \multicolumn{3}{|c|}{ Beitragszahlungen } & \multicolumn{3}{|c|}{ Rückflüsse-Agrarfonds } & \multicolumn{3}{|c|}{$\begin{array}{l}\text { Rückflüsse- } \\
\text { Strukturfonds }\end{array}$} \\
\hline & Basis & erh.-1 & em.-2 & Basis & red.-1 & Red.-2 & Basis & red.-1 & red.-2 \\
\hline Belgien & 1.369 & 1.594 & 1.817 & 0.453 & 0.326 & 0.222 & 1.413 & 1.012 & 0.739 \\
\hline Dänemark & 1.099 & 1.279 & 1.458 & 0.902 & 0.650 & 442 & 0.247 & 0.177 & 0.129 \\
\hline D eutschland & 1.154 & 1.344 & 1.532 & 0.314 & 0.226 & 154 & 0.245 & 0.175 & 0.128 \\
\hline Finnland & 1.043 & 1.214 & 1.384 & 0.561 & 0.404 & 0.275 & 0.474 & 0.339 & 0.248 \\
\hline Frankreich & 1.083 & 1.261 & 1.437 & 0.752 & 0.542 & 0.369 & 0.268 & 0.191 & 0.140 \\
\hline G riechenland & 1.112 & 1.295 & 1.476 & 2.577 & 1.857 & 1.265 & 2.661 & 1.905 & 1.392 \\
\hline Großbritannien & 0.787 & 0.916 & 1.044 & 0.388 & 0.279 & 0.190 & 0.241 & 0.172 & 0.126 \\
\hline Irland & 1.243 & 1.447 & 1.649 & 3.680 & 2.651 & 1.805 & 2.406 & 1.722 & 1.259 \\
\hline Italien & 0.865 & 1.007 & 1.148 & 0.508 & 0.366 & 0.249 & 0.351 & 0.251 & 0.184 \\
\hline Luxemburg & 1.151 & 1.340 & 1.528 & 0.154 & 0.111 & 0.075 & 5.891 & 4.217 & 3.082 \\
\hline Niederlande & 1.519 & 1.768 & 2.015 & 0.552 & 0.397 & 0.271 & 0.252 & 0.181 & 0.132 \\
\hline Ö sterreich & 1.163 & 1.354 & 1.543 & 0.475 & 0.342 & 0.233 & 0.250 & 0.179 & 0.131 \\
\hline Portugal & 1.237 & 1.440 & 1.642 & 0.754 & 0.543 & 0.370 & 3.608 & 2.583 & 1.888 \\
\hline Schweden & 1.209 & 1.407 & 1.604 & 0.388 & 0.280 & 0.190 & 0.194 & 0.139 & 0.102 \\
\hline Spanien & 1.153 & 1.343 & 1.530 & 0.990 & 0.713 & 0.486 & 1.439 & 1.030 & 0.753 \\
\hline EU15 & 1.146 & 1.334 & 1.520 & 0.896 & 0.646 & 0.440 & 1.329 & 0.952 & 0.695 \\
\hline Tschechis & 1.280 & 1.491 & 1.699 & 1.731 & 1.247 & 0.849 & 3.371 & 2.413 & 1.764 \\
\hline Ungarn & 1.280 & 1.489 & 1.697 & 3.749 & 2.701 & 1.839 & 5.189 & 3.714 & 2.715 \\
\hline Polen & 1.280 & 1.490 & 1.699 & 2.620 & 1.887 & 1.285 & 5.420 & 3.880 & 2.836 \\
\hline Slowenien & 1.280 & 1.493 & 1.702 & 1.619 & 1.166 & 0.794 & 3.611 & 2.585 & 1.889 \\
\hline Estland & 1.280 & 1.493 & 1.701 & 2.953 & 2.127 & 1.449 & 5.487 & 3.928 & 2.871 \\
\hline Bulgarien & 1.280 & * & 1.701 & 3.320 & $*$ & 1.629 & 6.910 & $*$ & 3.615 \\
\hline Rumänien & 1.280 & * & 1.700 & 9.940 & $*$ & 4.877 & 15.743 & * & 8.236 \\
\hline Slowakei & 1.280 & * & 1.698 & 2.252 & $*$ & 1.105 & 9.958 & $*$ & 5.210 \\
\hline Lettland & 1.280 & * & 1.703 & 3.406 & $*$ & 1.671 & 5.804 & $*$ & 3.036 \\
\hline Litauen & 1.280 & 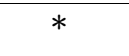 & 1.702 & 3.954 & * & 1.940 & 7.419 & $*$ & 3.882 \\
\hline MOEL & 1.280 & 1.491 & 1.700 & 3.554 & 1.826 & 1.744 & 6.891 & 3.304 & 3.605 \\
\hline Defizit-1 & 0.184 & 0.000 & 0.000 & 0.184 & 0.000 & 0.000 & 0.184 & 0.000 & 0.000 \\
\hline Defizit-2 & 0.370 & 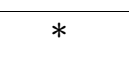 & 0.000 & 0.370 & $*$ & 0.000 & 0.370 & $*$ & 0.000 \\
\hline
\end{tabular}

Basis: Beitragssätze bzw. Rückflußsätze für die EU15-Länder für 1997 gemäß European Commission (1997,1998), die Beitragssätze und Rückflußsätze für MOEL gemäß Schätzungen von Breuss (1995) und Breuss \& Schebeck (1996). Es ergeben sich Defizite bei Erweiterung auf MOEL1-Länder (Defizit-1) bzw. MOEL2-Länder (Defizit-2), jeweils in \% des EU15-BIP. Zur Bedeutung von MOEL1 und MOEL2, siehe Legende zur Tabelle 2.

Die Spalten erh.-1 bzw. erh.-2 geben proportional angepaßte Beitragszahlungen, die das Budget einer auf die MOEL1Länder bzw. die MOEL1 plus die MOEL2-Länder erweiterten Union zum Ausgleich brächten. Analoges gilt für die Reduktion der Rückflüsse in den Spalten red.-1 bzw. red.-2. Die Zeilen E U15 bzw. MOEL weisen jeweils entsprechende Durchschnitte (arithmetisch) aus. 


\section{A bbildung 2 \\ Fiskalische Belastung und Integrationseffekte der Osterweitenung (MOE L1) für die Länder der E U 15}

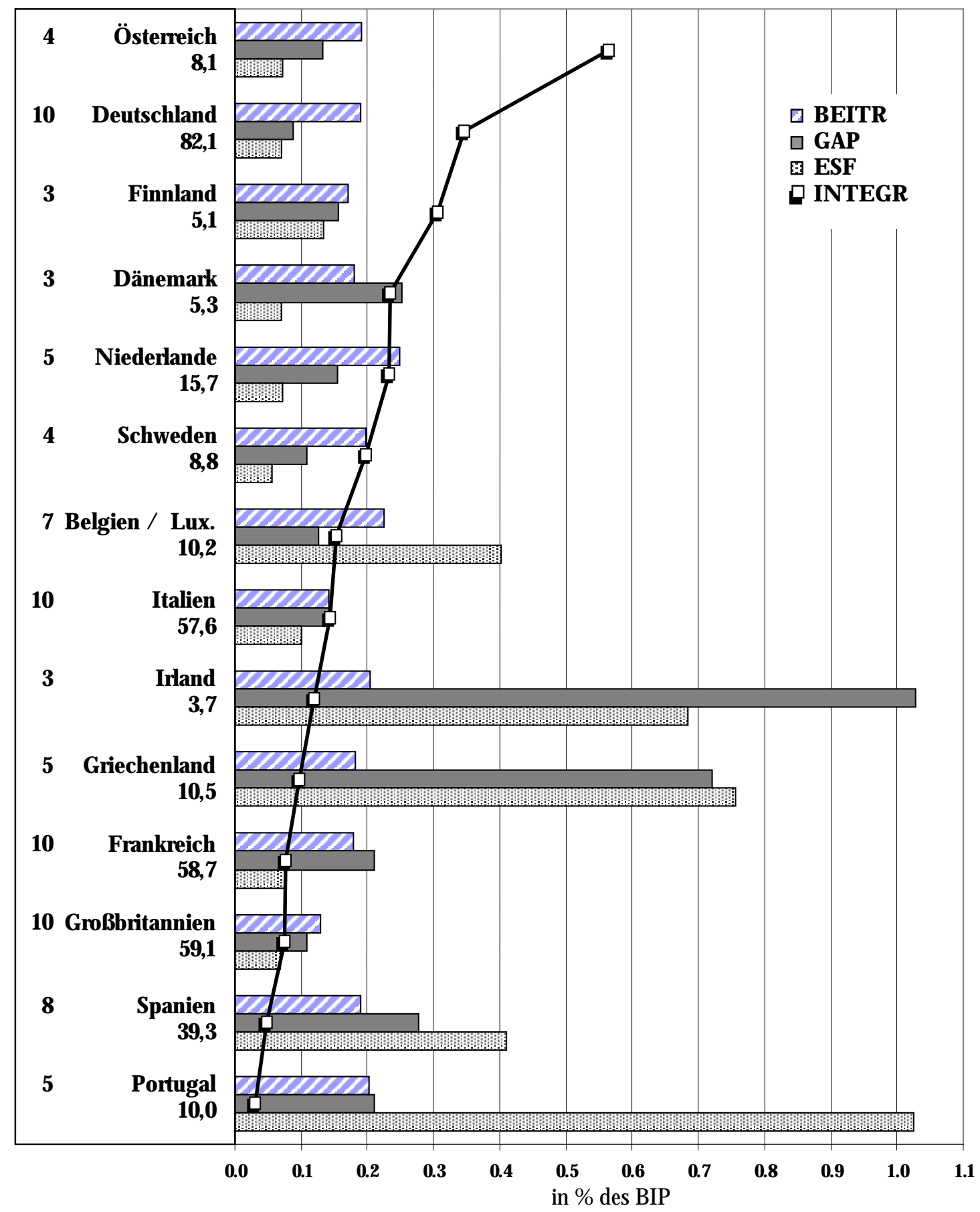

Zur Bedeutung von MOEL1, siehe Tabelle 2.

BE ITR, GAP und ESF : fiskalische Belastung bei alternativen Finanzierungsszenarien, errechnet aus Tabelle 3.

INTEGR: Gesamtwohlstandsgewinn aus G ütermarktintegration, siehe Text.

Die Ziffern links von den Ländernamen geben die Anzahl der Stimmen im Europäischen Rat an, unter den Ländernamen findet sich jeweils die Bevölkerung (in Millionen).

Quellen: http:/ / europa.eu.int/ en/ comm/ eurostat/ facts/ wwwroot/ de/ index.htm (Bevölkerung) http:/ / europa.eu.int/ inst/ en/ cl.htm (Stimmen im Rat) 